\title{
Brief review: Anesthetic neurotoxicity in the elderly, cognitive dysfunction and Alzheimer's disease
}

\section{Article de synthèse court: La neurotoxicité de l'anesthésie chez le patient âgé, le dysfonctionnement cognitif et la maladie d'Alzheimer}

\author{
Edward A. Bittner, MD • Yun Yue, MD • \\ Zhongcong Xie, MD \\ Received: 25 August 2010/Accepted: 26 October 2010/Published online: 21 December 2010 \\ (C) Canadian Anesthesiologists' Society 2010
}

\begin{abstract}
Purpose Postoperative cognitive decline in the elderly has emerged as a major health concern. In addition, there is a growing interest in the potential relationship between general anesthetic exposure and the onset and progression of Alzheimer's disease (AD). The available evidence of a possible association between anesthesia, surgery, and long-term cognitive effects, including $A D$, deserves consideration. In this review, we summarize the evidence for anesthesia-induced neurotoxicity in the elderly, while highlighting the limitations of existing data, and we put the literature into perspective for the clinician.

Principal findings A growing body of evidence suggests that general anesthetics may be neurotoxic to both young and aging brains. Much of the evidence originates from in vitro and in vivo studies with cells, rodents, and nonhuman
\end{abstract}

This article is accompanied by an editorial. Please see Can J Anesth 2011; 58(2).

\section{E. A. Bittner, MD}

Department of Anesthesia, Critical Care and Pain Medicine,

Massachusetts General Hospital and Harvard Medical School,

Boston, MA, USA

\section{Y. Yue, MD}

Department of Anesthesia, Beijing Chaoyang Hospital, Capital Medical University, Beijing, PR China

\section{Z. Xie, MD (ه)}

Geriatric Anesthesia Research Unit, Department of Anesthesia, Critical Care and Pain Medicine, Massachusetts General

Hospital and Harvard Medical School, 114 16th St., 3750, Charlestown, MA 02129-2060, USA

e-mail: zxie@partners.org primates. Despite the animal data suggesting a relationship between anesthesia and neurotoxicity in the elderly, a definitive link remains elusive in humans.

Conclusions The possible relation between anesthetic neurotoxicity, postoperative cognitive dysfunction, and $A D$ remains elusive. It remains unclear whether postoperative cognitive decline in the elderly is related more to perioperative stress and related medical co-morbidities.

\section{Résumé}

Objectif Le déclin cognitif postopératoire chez le patient âgé est aujourd'hui un problème de santé publique majeur. De plus, la relation potentielle entre l'exposition à un anesthésique général et l'apparition et l'évolution de la maladie d'Alzheimer (MA) fait l'objet de plus en plus de recherches. Les données probantes disponibles portant sur une association possible entre l'anesthésie, la chirurgie, et des effets cognitifs à long terme, y compris la MA, méritent d'être examinées. Dans cet article de synthèse, nous résumons les données probantes rapportant des cas de neurotoxicité induite par l'anesthésie chez le patient âgé, tout en mettant en exergue les limites des données existantes, et nous mettons en perspective la littérature à l'intention du clinicien.

Constatations principales Un nombre croissant de données probantes suggèrent que les anesthésiques généraux pourraient avoir des effets neurotoxiques sur le cerveau, qu'il s'agisse d'un patient jeune ou âgé. La plupart de ces données sont tirées d'études in vitro et in vivo réalisées sur des cellules, des rongeurs et des primates non humains. Malgré les données animales suggérant une relation entre l'anesthésie et la neurotoxicité chez le patient âgé, il est difficile d'établir un lien incontestable chez l'humain. 
Conclusion La relation possible entre la neurotoxicité liée à l'anesthésie, le dysfonctionnement cognitif postopératoire et la MA reste à établir clairement. À l'heure actuelle, il est difficile de déterminer si le déclin cognitif postopératoire chez le patient âgé n'est pas davantage lié au stress périopératoire et aux comorbidités médicales associées qu'à l'anesthésie.

Due to their lipid solubility, general anesthetics enter the brain readily in high concentrations, dissolving into cellular membranes, penetrating organelle, and acting on multiple receptors, ion channels, second messenger systems, and cytoskeletal components. ${ }^{1}$ Until recently, it has been assumed that these effects are reversible and nontoxic. However, a growing body of laboratory evidence suggests that general anesthetics may be neurotoxic to both young and aging brains. The evidence comes from in vitro and in vivo studies with cells, rodents, and nonhuman primates. ${ }^{2}$ Postoperative cognitive decline in the elderly has emerged as a major health concern. ${ }^{3}$ In addition, there is a growing interest in the potential relationship between general anesthetic exposure and the onset and progression of Alzheimer's disease (AD). ${ }^{4}$

In this review, we focus on the recent studies of anesthesia-induced neurotoxicity, while highlighting the limitations of existing data, and we put the literature into perspective for the clinician. Although this review focuses primarily on studies of anesthesia-induced neurotoxicity, it must be emphasized that many other studies have shown that anesthetics do not induce toxic effects; furthermore, some anesthetics have been reported to have organ (e.g., heart and brain) protective effects. Since anesthesiainduced protective effects are beyond the scope of this manuscript, we do not describe other deserving research results in this focused review.

\section{Anesthetic neurotoxicity}

Neurotoxicity is defined as structural or functional alteration in the nervous system resulting from exposure to a chemical, biological, or physical agent. ${ }^{5}$ The brain is especially vulnerable to the effects of neurotoxins at the extremes of age. The aged brain suffers from loss of neurons, reduced rates of neurogenesis, and synaptogenesis and accumulation of potentially toxic byproducts. Thus, reduced reserve and increased vulnerability lead to functional impairment from neural insults, such as oxidative stress or toxin exposure. Current hypotheses attribute the neurotoxic effects of volatile anesthetics to direct toxic effects (e.g., via altered calcium homeostasis), ${ }^{6}$ enhancement of endogenous neurodegenerative mechanisms (e.g., increased production of $\mathrm{A} \beta),{ }^{7}$ neuroinflammation triggered by surgically-induced systemic inflammation, ${ }^{8}$ or age-sensitive suppression of stem cell proliferation or differentiation. ${ }^{9}$

The study of neurotoxic effects of anesthetics in humans is limited by a number of factors, including difficulty in obtaining tissue for histological study and the confounding effects of surgery and associated diseases. To complicate matters, anesthetics may have neuroprotective effects depending on the drug, dose, and duration of exposure. ${ }^{10-14}$

There are a number of reasons for the delayed appreciation of the potential association between anesthetics and neurodegeneration, including the slowly progressive nature of neurodegenerative disease, limited follow-up by surgeons and anesthesiologists, presence of confounding factors, such as coincident illness, and the social stigma of the disease.

\section{Cognitive dysfunction in the elderly}

\section{Alzheimer's disease}

Alzheimer's disease is a neurodegenerative disease. It is the most common form of dementia in adults, affecting 35 million people worldwide and 5.3 million people in the United States alone. ${ }^{15}$ Alzheimer's disease is associated with significant morbidity and mortality and leads to death within three to nine years after diagnosis. ${ }^{15}$ Furthermore, $\mathrm{AD}$ has a major social and economic impact, since the people affected not only lose their own productivity and independence but also require continual one-on-one care. Clinically, AD typically presents with subtle onset of memory loss followed by a slowly progressive dementia over a course of several years.

Pathologically, the brains of $\mathrm{AD}$ patients have gross diffuse atrophy of the cerebral cortex with the secondary enlargement of the ventricular system. Cerebral plaques laden with $\beta$-amyloid peptides are a pathologic feature of AD. The peptides coalesce into intermediate assemblies, or they can arrange themselves into sheets of $\beta$-amyloid $(\mathrm{A} \beta)$ fibrils that can form advanced amyloid plaques. This excess accumulation of $\mathrm{A} \beta$ may be the initiating factor in $\mathrm{AD}$ ("amyloid hypothesis"). ${ }^{16}$ Like $\mathrm{A} \beta$, abnormal tau molecules are cytotoxic and contribute to dementia. Abnormal tau and $\mathrm{A} \beta$ appear to contribute to synaptic loss and dysfunction even before tangles and plaques are detected. ${ }^{17,18}$ In addition, a number of molecular lesions have been identified in $\mathrm{AD}$, with most centering on a few cellular pathways. Common themes include accumulation of abnormal proteins resulting in inflammatory and oxidative damage and ultimately resulting in synaptic loss. 
The principal risk factor for $\mathrm{AD}$ is age. A number of etiologic factors have been proposed to contribute to the neuropathogenesis of $\mathrm{AD}$, including genetic susceptibility, metabolic alterations, and environmental exposures. Identification of several $\mathrm{AD}$ associated genes has provided a foundation for understanding the pathogenesis of $\mathrm{AD}$. However, the majority of cases are sporadic in origin, and the exact causes of sporadic AD are unknown.

\section{Postoperative cognitive dysfunction}

Many elderly patients experience difficulties with memory, concentration and attention after surgery and anesthesia, which is referred to as postoperative cognitive dysfunction (POCD). These cognitive changes are usually short-lived with normal function returning within a few days, but changes may persist for weeks or more. POCD is often mild and only detected by neuropsychological testing after anesthesia and surgery. In the extreme, it may manifest clinically as memory loss, impaired higher level cognitive function, psychomotor derangement, difficulties with fine motor coordination, dementia, delirium, or depression. ${ }^{19}$ Postoperative cognitive dysfunction has been associated with leaving the labour market prematurely, increased dependency on social support, and increased mortality. ${ }^{20}$

The incidence of POCD after noncardiac surgery has been reported to be as high as $26 \%$ at one week, $10 \%$ at three months, ${ }^{21} 5 \%$ at six months, and $1 \%$ at 12 months after surgery. ${ }^{22}$ However, the incidence of POCD after cardiac surgery can be as high as 53\%, 36\%, 24\%, and $42 \%$ at discharge, six weeks, six months, and five years, respectively, after surgery. ${ }^{23}$ It should be emphasized that the definition of POCD is still in evolution ${ }^{24}$; therefore, the difference in POCD incidence in these studies might be due to different criteria used in determining POCD.

The mechanism of POCD is unclear. However, since $\mathrm{A} \beta$ is a peptide naturally present in the central nervous system, with higher levels in the elderly, there has been speculation that these higher amounts of $\mathrm{A} \beta$ in the aging brain are available to interact with general anesthetics and other perioperative factors leading to deleterious effects on the brain and increasing the risk for POCD.

\section{Evidence for anesthesia-mediated neurodegeneration}

Cell and tissue culture

Inhaled anesthetics have been shown to induce damage in a variety of tissues and cells, including hippocampal slices, neuroglioma cells, and primary cortical and striatal neurons. The various in vitro models have shown different toxicity to inhaled anesthetics. Cells transfected with genes associated with $\mathrm{AD}$ have been shown to be more sensitive to anesthetic toxicity. ${ }^{25}$ As recently reviewed in Tang et al., in vitro studies using isolated proteins, cells in culture, and brain slices have provided evidence that the inhaled anesthetics may produce effects consistent with increased cellular stress through recognized pathways of neurodegeneration $^{2}$ Most of this work has been done with the commonly used inhalation anesthetics, isoflurane, sevoflurane, and desflurane. Isoflurane, ${ }^{26-28}$ sevoflurane, ${ }^{29}$ desflurane plus hypoxia, ${ }^{30}$ and isoflurane plus nitrous oxide $^{31}$ have been shown to induce cellular apoptosis and increase $\mathrm{A} \beta$ generation. It is important to note that most of these studies were performed with tumour cells rather than with primary neurons.

Animal models

Animal models allow the exploration of mechanisms by which anesthetics may induce AD pathogenesis as well as provide a way to examine the effects of anesthesia and surgery independently. Several studies from animal models suggest that anesthetic exposure impairs cognitive function and increases pathology commonly associated with $\mathrm{AD}$. In adult wild-type rats and mice, exposure to isoflurane can result in decrements in learning that persists for weeks or months. ${ }^{32-35}$ Furthermore, in the elderly rodent, exposure to isoflurane, nitrous oxide, or the combination of isofluranenitrous oxide can cause persistent memory impairment. ${ }^{32-36}$ Anesthesia with $1.4 \%$ isoflurane ${ }^{28}$ or $2.5 \%$ sevoflurane $^{29}$ for two hours has been shown to induce caspase- 3 activation and increase $\mathrm{A} \beta$ levels in brain tissues of five-month-old wildtype mice. It should be noted that these animals received isoflurane or sevoflurane plus $100 \%$ oxygen. Therefore, the possibility cannot be excluded that the combination of anesthetics and high oxygen concentrations induces neurotoxicity rather than anesthetic agents alone. These studies did not assess the potential impairment of cognitive function associated with the biochemical changes.

A recent study reported that anesthesia with $2.1 \%$ sevoflurane for six hours can induce caspase- 3 activation and increase $\mathrm{A} \beta$ levels in the brain tissues of neonatal mice (6 days old) and AD transgenic mice. ${ }^{37}$ Furthermore, the sevoflurane anesthesia led to a greater degree of neurotoxicity in the brain tissues of the $\mathrm{AD}$ transgenic mice when compared with neonatal mice with increased tumor necrosis factor-alpha levels in the brain tissues of the $\mathrm{AD}$ transgenic mice. Early studies have shown that hypothermia $^{38}$ and anesthesia ${ }^{39}$ can induce tau hyperphosphorylation. In the transgenic mouse models of $\mathrm{AD}$, increased $\mathrm{A} \beta$ plaque loads and tau aggregation have been demonstrated after halothane and isoflurane exposure, respectively. ${ }^{25}$ In a mouse model, Run et al. found that anesthesia, even for short periods of time (30 sec to five 
minutes), resulted in tau phosphorylation in specific AD-related hyperphosphorylation sites. ${ }^{40}$

Other studies have suggested that the link between inhaled anesthetics and neurodegenerative changes is dose and context specific. In a study to investigate the effects of repeated isoflurane exposure (35 min daily for four days) on juvenile and mature rodents, Zhu et al. found that recognition and reversal learning were impaired in the young rodents, and these deficits became more pronounced as the animals grew older. ${ }^{41}$ The memory deficit was paralleled by a reduction in the hippocampal stem cell pool and reduced neurogenesis. Interestingly, the mature animals were unaffected by the isoflurane exposure. In another laboratory, Stratmann et al. examined hippocampal progenitor proliferation, neuronal lineage selection and survival, as well as learning and spatial memory acquisition in seven-day-old and 60-day-old rats. The authors found a delayed onset, progressive and persistent deficit in memory tasks in the seven-day-old rats, whereas the 60-day-old rats showed improvement in spatial reference memory. Furthermore, there was an isoflurane-induced decrease in neuronal progenitor proliferation in the seven-day-old rats and an increase in neuronal differentiation in the 60-dayold rats. ${ }^{42}$ In another study, the same group of investigators examined aged rats exposed to one minimum alveolar concentration of isoflurane for four hours. The investigators failed to detect a difference in hippocampal progenitor proliferation or in neuronal survival compared with agematched controls. In addition, no difference was found in associative learning, spatial memory acquisition, or retention four months after isoflurane exposure. ${ }^{43}$

Given these conflicting results, it remains unclear as to the conditions under which volatile anesthetic exposure results in neurodegenerative changes and cognitive impairment. Although animal models have provided some insight into the mechanisms of neuronal loss and accumulation of neurotoxic proteins, the connection between these lesions, symptoms, and causes of the disease remains difficult to unravel. A causal link between anestheticinduced biochemical markers of neurotoxicity and the development of long-term cognitive dysfunction is required to solidify the proposed link between inhaled anesthetics and neurodegenerative changes.

Surgery itself may be a risk factor for cognitive dysfunction. Wan et al. subjected anesthetized rats to splenectomy vs no surgery and demonstrated that there was a period of cognitive dysfunction in the splenectomized rats, as assessed by performance in a Y-maze, which was associated with biochemical markers of hippocampal glial cell activation and inflammation. ${ }^{8}$ In another recent study, Wan et al. showed that partial hepatectomy under general anesthesia in 16-month-old mice induced learning and memory impairment as demonstrated by Morris Water Maze performance as well as gliosis, $\mathrm{A} \beta$ accumulation, and tau protein phosphorylation as compared with anesthetized controls. ${ }^{44}$ Interestingly, the anesthetized rats and mice in these studies that did not undergo surgery showed none of the changes in maze performance or inflammatory markers, suggesting that observed changes were not related to anesthesia alone. In addition, the anesthesia used in these studies was fentanyl plus droperidol ${ }^{8}$ and chloral hydrate. ${ }^{44}$ Therefore, it remains to be determined whether the volatile anesthetics (e.g., isoflurane) that have been shown to induce apoptosis, $\mathrm{A} \beta$ accumulation, and tau phosphorylation can lead to learning and memory impairment in the same rodent model of surgery vs no surgery. Given that surgery without general anesthesia can also cause POCD in humans, ${ }^{45,46}$ it is important to determine the effects of surgery without general anesthesia on the cognitive changes.

\section{Evidence for an association between volatile anesthetic exposure and neurotoxicity in humans}

Despite the animal data suggesting a relationship between anesthesia and AD neuropathogenesis, a definitive link remains elusive in humans. Several small human studies have examined the link between anesthesia, surgery, and AD with conflicting results. A case control study by Gasparini et al. found no association between $\mathrm{AD}$ and exposure to anesthesia in the one to five years preceding disease onset, nor between the risk of $\mathrm{AD}$ and the number of surgical operations. ${ }^{47}$ Similarly, Bohnen et al. found no significant difference in mean cumulative duration of exposure to general anesthesia and increased risk of AD. ${ }^{48}$ However in another retrospective study, Bohnen et al. found an inverse relationship between age of onset of $\mathrm{AD}$ and cumulative anesthesia exposure before the age of 50, which suggests that the manifestation of dementia in AD patients may be related to anesthetic exposure at an earlier age. $^{49}$ In a cohort analysis of patients undergoing coronary artery bypass graft (CABG) or percutaneous transluminal coronary angioplasty (PTCA), an increased risk for emergence of $\mathrm{AD}$ was found in patients undergoing $\mathrm{CABG}$, suggesting that cardiac surgery and anesthesia may be associated with long-term cognitive effects in an aging population with reduced neuronal reserve. ${ }^{50}$

Cognitive dysfunction is well-documented after cardiac surgery, and cardiopulmonary bypass has been implicated as a risk factor for cognitive decline and dementia. ${ }^{51}$ However, even in this patient population, the results are contradictory. In a population-based case-control study, Knopman et al. found no association between CABG and risk for dementia. ${ }^{52}$ In patients undergoing cardiac surgery, multiple risk factors have been implicated in the development of POCD, including hypoperfusion, cerebral 
embolism, atrial fibrillation, myocardial ischemia, systemic inflammatory response, and pre-existing cerebral injury or cognitive impairment. These differing cited causes could predispose or exacerbate vulnerability to cognitive impairment without invoking anesthetic neurotoxicity as a culprit.

A recent study examining the impact of non-cardiac surgery, illness, or neither on cognitive decline in the elderly did not detect long-term cognitive decline independently attributable to surgery or illness, nor were these events associated with decline to dementia, although demented patients declined more markedly than nondemented patients. ${ }^{53}$ However, as with all other retrospective studies, this study has several limitations, including difficulty in finding appropriate controls, as described in the manuscript.

A growing body of literature describes an association between critical illness and cognitive impairment. ${ }^{54-56}$ In a recent cohort study of older adults without dementia at baseline, Ehlenbach et al. found that those who were hospitalized for acute care or critical illness had a greater likelihood of cognitive decline compared with those who were not hospitalized. ${ }^{57}$ Furthermore, hospitalization for noncritical illness was also significantly associated with the development of dementia. The findings from these studies strongly suggest the need for adequately powered multicentre clinical trials to define in further detail the interplay of anesthesia, surgery, and intercurrent illness on the development of cognitive dysfunction in the elderly.

\section{Do we need to change clinical practice?}

The available evidence suggests a possible association between anesthesia, surgery, and long-term cognitive effects, including AD. Nevertheless, it is premature to alter clinical practice because three issues have not been evaluated rigorously in clinical trials. To date, no study has shown that general anesthetics administered at clinically relevant doses and for clinically relevant durations cause neurotoxicity in humans. There is no scientific basis for either recommending or contraindicating specific anesthetic agents or techniques on the basis of neurotoxicity or risk of cognitive morbidity in the elderly. ${ }^{58}$ It is essential to bear in mind that the overriding goals of anesthesia are to ensure patient comfort, patient safety, and optimal conditions for surgery. We should not compromise the well-established benefits of general anesthesia because of some early laboratory results without substantiated clinical relevance.

So what steps should the clinician consider? It seems rational to minimize the complexity of anesthetic regimens and to use the lowest reasonable effective doses and concentrations of anesthetic required according to individual patient needs, the varying levels of surgical stimulation, and postoperative analgesic requirements. However, this may be difficult, since the minimal alveolar concentration values for animal models of $\mathrm{AD}$ are increased relative to their non- $\mathrm{AD}$ counterparts. ${ }^{59,60}$ Although a recent study did not find an association between depth of anesthesia and development of POCD,${ }^{61}$ further research is certainly needed. Use of regional anesthesia instead of general anesthesia has been suggested to avoid volatile anesthetic exposure. However, there is no solid evidence to show that the incidence of POCD is different after general anesthesia as compared with regional anesthesia. ${ }^{62}$

Rank ordering for amyloidogenic potential and cytotoxicity has been reported for inhalational anesthetics in cell culture. ${ }^{26,63}$ Isoflurane has greater potency than either sevoflurane or desflurane to cause calcium release from the endoplasmic reticulum and to induce cell damage. However, a recent randomized trial comparing desflurane with sevoflurane in elderly patients found no difference in the incidence of POCD, although performances in several cognitive tests favoured desflurane. ${ }^{64}$ Although tempting to favour the use of the shorter-acting volatile anesthetics for this reason, the literature does not allow definitive conclusions to be drawn at this time. Hypothermia causes tau hyperphosphorylation and associated memory impairment in animals. ${ }^{38,65}$ Given the involvement of tau hyperphosphorylation in $\mathrm{AD}$, it provides another reason to ensure normothermia in the perioperative period.

\section{Areas for further study}

Given the available evidence, a possible association between anesthesia, surgery, and long-term cognitive effects, including AD, needs to be taken seriously. There is a great need for further well-designed adequately powered randomized trials and observational studies to assess the impact of anesthetic exposure on cognitive decline. Since surgery itself may be a contributing factor, the need for human studies evaluating anesthesia exposure in the absence of surgery would be particularly informative.

There are five key questions that should direct research efforts:

1. What is the likelihood and magnitude of risk?

2. At what age (if any) does the risk of exposure become significant?

3. Are there patient factors other than age that influence risk?

4. To what extent do different anesthetic techniques and agents impact risk?

5. Are there protective agents that can be used?

Interrogating large pre-existing databases with many years of individual patient data (e.g., Veterans Affairs 
Medicare) seems like the most rapid and risk-free way of examining the association between anesthesia, surgery, and postoperative cognitive decline. Biomarkers derived from structural and functional neuroimaging studies and those measured in cerebrospinal fluid, show great promise for identifying preclinical pathology and for monitoring change over time and after anesthetic exposure. ${ }^{66,67}$ Since adequately powered clinical trials examining these questions will require many years to conduct and to analyze, there is an immediate need for further animal studies to define the effects of both anesthesia and surgery on the pathogenesis of $\mathrm{AD}$. These studies will complement ongoing clinical trials by establishing mechanistic hypotheses, vulnerable windows, and less provocative anesthetics.

In summary, it will take time to examine the potential link between the laboratory findings suggesting anestheticinduced neurotoxicity and adverse clinical outcomes. The possible relation between anesthetic neurotoxicity, POCD, and $\mathrm{AD}$ remains elusive. It remains unclear whether postoperative cognitive decline is related more to perioperative stress and the presence of patient-associated co-morbidities than to the effects of anesthetic exposure. It is important that the perioperative physician is aware of the potential link between anesthesia, surgery, and cognitive decline in the elderly, because the perioperative setting provides a unique opportunity to evaluate pre-emptive therapies. As clinicians, it is important that we remain abreast of the ongoing research in this important field of investigation.

Funding sources Several studies referenced in this review were supported by National Institutes of Health grants (Bethesda, Maryland) (K08 NS048140, R21 AG029856, and R01 GM088801); American Geriatrics Society Jahnigen Award (New York, NY); Investigator-Initiated Research Grant from Alzheimer's Association (Chicago, IL) to Dr. Zhongcong Xie.

Competing interests None declared.

\section{References}

1. Campagna JA, Miller KW, Forman SA. Mechanisms of actions of inhaled anesthetics. N Engl J Med 2003; 348: 2110-24.

2. Tang J, Eckenhoff $M F$, Eckenhoff $R G$. Anesthesia and the old brain. Anesth Analg 2010; 110: 421-6.

3. Fodale V, Santamaria LB, Schifilliti, Mandel PK. Anaesthetics and postoperative cognitive dysfunction: a pathological mechanism mimicking Alzheimer's disease. Anaesthesia 2010; 65: 38895.

4. Baranov D, Bickler PE, Crosby GJ, et al. Consensus statement: First International Workshop on Anesthetics and Alzheimers disease. Anesth Analg 2009; 108: 1627-30.

5. Costa LG, Ascher M, Vitalone A, Syversen T, Soldin OP. Developmental neuropathology of environmental agents. Annu Rev Phamacol Toxicol 2004; 44: 87-110.
6. Wei H, Xie Z. Anesthesia, calcium homeostasis and Alzhemiers disease. Curr Alzheimer Res 2009; 6: 30-5.

7. Xie Z, Dong $Y$, Maeda U, et al. The common inhalation anesthetic isoflurane induces apoptosis and increases amyloid beta protein levels. Anesthesiology 2006; 104: 988-94.

8. Wan $Y, X u J$, Ma D, Zeng Y, Cibelli M, Maze M. Postoperative impairment of cognitive function in rats: a possible role for cytokine-mediated inflammation in the hippocampus. Anesthesiology 2007; 106: 436-43.

9. Sall JW, Stratmann $G$, Leong $J$, et al. Isoflurane inhibits growth but does not cause cell death in hippocampal neural precursor cells grown in culture. Anesthesiology 2009; 110: 826-33.

10. Li L, Peng L, Zuo Z. Isoflurane preconditioning increases B-cell lymphoma-2 expression and reduces cytochrome $\mathrm{c}$ release from the mitochondria in the ischemic penumbra of rat brain. Eur $\mathbf{J}$ Pharmacol 2008; 586: 106-13.

11. Gray JJ, Bickler PE, Fahlman CS, Zhan X, Schuyler JA. Isoflurane neuroprotection in hypoxic hippocampal slice cultures involves increases in intracellular $\mathrm{Ca} 2+$ and mitogen-activated protein kinases. Anesthesiology 2005; 102: 606-15.

12. Codaccioni JL, Velly LJ, Moubarik C, Bruder NJ, Pisano PS, Guillet BA. Sevoflurane preconditioning against focal cerebral ischemia: inhibition of apoptosis in the face of transient improvement of neurological outcome. Anesthesiology 2009; 110: 1271-8.

13. Wei H, Liang G, Yang $H$. Isoflurane preconditioning inhibited isoflurane-induced neurotoxicity. Neurosci Lett 2007; 425: 59-62.

14. Zhu J, Jiang X, Shi E, Ma H, Wang J. Sevoflurane preconditioning reverses impairment of hippocampal long-term potentiation induced by myocardial ischaemia-reperfusion injury. Eur J Anaesthesiol 2009; 26: 961-8.

15. Querfurth HW, LaFerla FM. Alzheimer's disease. N Engl J Med 2010; 324: 329-44.

16. Tanzi RE, Bertram L. Twenty years of the Alzheimer's disease amyloid hypothesis: a genetic perspective. Cell 2005; 120: 54555.

17. Tanzi RE. The synaptic Abeta hypothesis of Alzheimer disease. Nat Neurosci 2005; 8: 977-9.

18. Tanzi RE. Tangles and neurodegenerative disease: a surprising twist. N Engl J Med 2005; 353: 1853-5.

19. Ramaiah $R$, Lam AM. Postoperative cognitive dysfunction in the elderly. Anesthesiol Clin 2009; 27: 485-96.

20. Steinmetz J, Christensen KB, Lund T, Lohse N, Rasmussen LS, ISPOCD Group. Long-term consequences of postoperative cognitive dysfunction. Anesthesiology 2009; 110: 548-55.

21. Moller JT, Cluitmans P, Rasmussen LS, et al. Long-term postoperative cognitive dysfunction in the elderly ISPOCD1 study. ISPOCD investigators. International Study of Post-Operative Cognitive Dysfunction. Lancet 1998; 351: 857-61.

22. Abildstrom H, Rasmussen LS, Rentowl P, et al. Cognitive dysfunction 1-2 years after non-cardiac surgery in the elderly. ISPOCD group. International Study of Post-Operative Cognitive Dysfunction. Acta Anaesthesiol Scand 2000; 44: 1246-51.

23. Newman MF, Kirchner JL, Phillips-Bute B, et al. Longitudinal assessment of neurocognitive function after coronary-artery bypass surgery. N Engl J Med 2001; 344: 395-402.

24. Rudolf JL, Schreiber KA, Culley DJ, et al. Measurement of postoperative cognitive dysfunction after cardiac surgery: a systemic review. Acta Anaesthesiol Scand 2010; 54: 663-77.

25. Bianchi SL, Tran T, Liu C, et al. Brain and behavior changes in 12-month-old $\mathrm{Tg} 2576$ and nontransgenic mice exposed to anesthetics. Neurobiol Aging 2008; 29: 1002-10.

26. Eckenhoff $R G$, Johansson JS, Wei H, et al. Inhaled anesthetic enhancement of amyloid-beta oligomerization and cytotoxicity. Anesthesiology 2004; 101: 703-9. 
27. Wei H, Liang $G$, Yang $H$, et al. The common inhalational anesthetic isoflurane induces apoptosis via activation of inositol 1,4,5trisphosphate receptors. Anesthesiology 2008; 108: 251-60.

28. Xie Z, Culley DJ, Dong $Y$, et al. The common inhalational anesthetic isoflurane induces caspase activation and increases amyloid beta-protein level in vivo. Ann Neurol 2008; 64: 618-27.

29. Dong $Y$, Zhang $G$, Zhang B, et al. The common inhalational anesthetic sevoflurane induces apoptosis and increases betaamyloid protein levels. Arch Neurol 2009; 66: 620-31.

30. Zhang B, Dong $Y$, Zhang $G$, et al. The inhalation anesthetic desflurane induces caspase activation and increases amyloid betaprotein levels under hypoxic conditions. J Biol Chem 2008; 283: 11866-75.

31. Zhen $Y$, Dong $Y, W u X$, et al. Nitrous oxide plus isoflurane induces apoptosis and increases beta-amyloid protein levels. Anesthesiology 2009; 111: 741-52.

32. Culley DJ, Baxter M, Yukhananov R, Crosby G. The memory effects of general anesthesia persist for weeks in young and aged rats. Anesth Analg 2003; 96: 1004-9.

33. Culley DJ, Baxter MG, Crosby CA, Yukhananov R, Crosby G. Impaired acquisition of spatial memory 2 weeks after isoflurane and isoflurane-nitrous oxide anesthesia in aged rats. Anesth Analg 2004; 99: 1393-7.

34. Culley DJ, Baxter MG, Yukhananov R, Crosby G. Long-term impairment of acquisition of a spatial memory task following isoflurane-nitrous oxide anesthesia in rats. Anesthesiology 2004; 100: 309-14.

35. Crosby C, Culley DJ, Baxter MG, Yukhananov R, Crosby G. Spatial memory performance 2 weeks after general anesthesia in adult rats. Anesth Analg 2005; 101: 1389-92.

36. Culley DJ, Raghavan SV, Waly $M$, et al. Nitrous oxide decreases cortical methionine synthase transiently but produces lasting memory impairment in aged rats. Anesth Analg 2007; 105: 83-8.

37. Lu $Y$, Wu X, Dong $Y, X u Z$, Zhang $Y$, Xie Z. Anesthetic sevoflurane causes neurotoxicity differently in neonatal naïve and Alzheimer disease transgenic mice. Anesthesiology 2010; 112: 1404-16.

38. Planel E, Richter KE, Nolan CE, et al. Anesthesia leads to tau hyperphosphorylation through inhibition of phosphatase activity by hypothermia. J Neurosci 2007; 27: 3090-7.

39. Planel E, Bretteville A, Liu L, et al. Acceleration and persistence of neurofibrillary pathology in a mouse model of tauopathy following anesthesia. FASEB J 2009; 23: 2595-604.

40. Run X, Liang Z, Zhang L, Iqbal K, Grundke-Iqbal I, Gong CX. Anesthesia induces phosphorylation of tau. J Alzheimers Dis 2009; 16: 619-26.

41. Zhu C, Gao J, Karlsson N, et al. Isoflurane anesthesia induced persistent, progressive memory impairment, caused a loss of neural stem cells, and reduced neurogenesis in young, but not adult, rodents. J Cereb Blood Flow Metab 2010; 30: 1017-30.

42. Stratmann $G$, Sall JW, May LD, et al. Isoflurane differentially affects neurogenesis and long-term neurocognitive function in 60day-old and 7-day-old rats. Anesthesiology 2009; 110: 834-48.

43. Stratmann G, Sall JW, Bell JS, et al. Isoflurane does not affect brain cell death, hippocampal neurogenesis, or long-term neurocognitive outcome in aged rats. Anesthesiology 2010; 112: 305-15.

44. Wan $Y, X u J$, Meng $F$, et al. Cognitive decline following major surgery is associated with gliosis, beta-amyloid accumulation and tau phosphorylation in old mice. Crit Care Med 2010; DOI: 10.1097/CCM.0b013e3181f17bcb.

45. Williams-Russo P, Sharrock NE, Mattis S, Szatrowski TP, Charlson ME. Cognitive effects after epidural vs general anesthesia in older adults. A randomized trial. JAMA 1995; 274 : 44-50.

46. Rasmussen LS, Johnson T, Kuipers HM, et al. Does anaesthesia cause postoperative cognitive dysfunction? A randomised study of regional versus general anaesthesia in 438 elderly patients. Acta Anaesthesiol Scand 2003; 47: 260-6.

47. Gasparini M, Vanacore $N$, Schiaffini $C$, et al. A case-control study of Alzheimer's disease and exposure to anesthesia. Neurol Sci 2002; 23: 11-4.

48. Bohnen NI, Warner MA, Kokmen E, Beard CM, Kurland LT. Alzheimer's disease and cumulative exposure to anesthesia: a case-control study. J Am Geriatr Soc 1994; 42: 198-201.

49. Bohnen N, Warner MA, Kokmen E, Kurland LT. Early and midlife exposure to anesthesia and the age of onset of Alzheimer's disease. Int J Neurosci 1994; 77: 181-5.

50. Lee TA, Wolozin B, Weiss KB, Bednar MM. Assessment of the emergence of Alzheimer's disease following coronary artery bypass graft surgery or percutaneous transluminal coronary angioplasty. J Alzheimers Dis 2005; 7: 319-24.

51. Raja PV, Blumenthal JA, Doraiswamy PM. Cognitive deficits following coronary artery bypass grafting: prevalence, prognosis, and therapeutic strategies. CNS Spectr 2004; 9: 76372.

52. Knopman DS, Petersen RC, Cha RH, Edland SD, Rocca WA. Coronary artery bypass grafting is not a risk factor for dementia or Alzheimers disease. Neurology 2005; 65: 986-90.

53. ccAvidan MS, Searleman AC, Storandt M, et al. Long-term cognitive decline in older subjects was not attributable to noncardiac surgery or major illness. Anesthesiology 2009; 111: 964-70.

54. Hopkins RO, Weaver LK, Collingridge D, Parkinson RB, Chan $K J$, Orme JF Jr. Two-year cognitive, emotional, and quality-oflife outcomes in acute respiratory distress syndrome. Am J Respir Crit Care Med 2005; 171: 340-7.

55. Sukantarat KT, Burgess PW, Williamson RC, Brett SJ. Prolonged cognitive dysfunction in survivors of critical illness. Anaesthesia 2005; 60: 847-53.

56. Hopkins RO, Jackson JC. Long-term neurocognitive function after critical illness. Chest 2006; 130: 869-78.

57. Ehlenbach WJ, Hough CL, Crane PK, et al. Association between acute care and critical illness hospitalization and cognitive function in older adults. JAMA 2010; 303: 763-70.

58. Culley DJ, Xie Z, Crosby G. General anesthetic-induced neurotoxicity: an emerging problem for the young and old? Curr Opin Anaesthesiol 2007; 20: 408-13.

59. Eckel B, Richtsfeld M, Starker L, Blobner M. Transgenic Alzheimer mice have a larger minimum alveolar concentration of isoflurane than their non-transgenic littermates. Anesth Analg 2010; 110: 438-41.

60. Bianchi SL, Caltagarone BM, LaFerla FM, Eckenhoff RG, Kelz $M B$. Inhaled anesthetic potency in aged Alzheimer mice. Anesth Analg 2010; 110: 427-30.

61. Steinmetz J, Funder KS, Dahl BT, Rasmussen LS. Depth of anaesthesia and post-operative cognitive dysfunction. Acta Anaesthesiol Scand 2010; 54: 162-8.

62. Rasmussen LS. Postoperative cognitive dysfunction: incidence and prevention. Best Pract Res Clin Anaesthesiol 2006; 20: 31530.

63. Yang H, Liang G, Hawkins BJ, Madesh M, Pierwola A, Wei H. Inhalational anesthetics induce cell damage by disruption of intracellular calcium homeostasis with different potencies. Anesthesiology 2008; 109: 243-50.

64. Rortgen D, Kloos J, Fries $M$, et al. Comparison of early cognitive function and recovery after desflurane or sevoflurane anaesthesia in the elderly: a double-blinded randomized controlled trial. $\mathrm{Br} \mathrm{J}$ Anaesth 2010; 104: 167-74.

65. Tan W, Cao X, Wang J, Lv $H, W u B, M a H$. Tau hyperphosphorylation is associated with memory impairment after exposure to $1.5 \%$ isoflurane with temperature maintenace in rats. Eur J Anaesthesiol 2010; 27: 835-41. 
66. Hampel H, Burger $K$, Teipel SJ, Bokde AL, Zetterberg H, Blennow $K$. Core candidate neurochemical and imaging biomarkers of Alzheimer's disease. Alzheimers Dement 2008; 4: $38-48$.
67. van Rossum IA, Vos S, Handels R, Visser PJ. Biomarkers as predictors for conversion from mild cognitive impairment to Alzheimer-type dementia: implications for trial design. J Alzheimers Dis 2010; 20: 881-91. 